Resolution of the Klein paradox

This article has been downloaded from IOPscience. Please scroll down to see the full text article.

2011 Phys. Scr. 83025001

(http://iopscience.iop.org/1402-4896/83/2/025001)

View the table of contents for this issue, or go to the journal homepage for more

Download details:

IP Address: 143.106.18.141

The article was downloaded on 25/02/2011 at 13:47

Please note that terms and conditions apply. 


\title{
Resolution of the Klein paradox
}

\author{
A D Alhaidari \\ Saudi Center for Theoretical Physics, Jeddah, Saudi Arabia \\ E-mail: haidari@mailaps.org
}

Received 12 June 2010

Accepted for publication 30 November 2010

Published 14 January 2011

Online at stacks.iop.org/PhysScr/83/025001

\begin{abstract}
We present a resolution of the Klein paradox within the framework of one-particle relativistic quantum mechanics. Not only reflection becomes total but the vacuum remains neutral as well. This is accomplished by replacing the physical pair production process with virtual negative energy 'incidence' within the barrier in a similar manner to what is done with virtual sources in optics and image charges in electrostatics.
\end{abstract}

PACS numbers: 03.65.Pm, 03.65.Nk, 03.65.Ca

The physics and mathematics of the Dirac equation are very rich and illuminating. However, this is true only if one includes the complete solution space of the equation. It is well known that the Dirac equation has positive as well as negative energy solutions [1]. The negative energy solutions are the subject of various interpretations that wax and wane throughout the history of the equation. However, since the equation is linear, the complete solution must be a linear combination of the two. Physical and mathematical results and interpretations thereof are correct only if the full contribution of the complete solution is accounted for. Klein's paradox [2] results from the conventional solution of the Dirac equation for a potential step of height $V$ that is larger than $2 m$, where $m$ is the rest mass of the particle $(\hbar=c=1)$. If the energy of the particle is in the range $+m<E<V-m$ (known as the Klein energy zone), then partial reflection and not total reflection will result although the energy is lower than the height of the barrier. We will show that this paradox results from eliminating part of the negative energy solution under the potential step without accounting for the physical pair production process. We also give a proper interpretation of these solutions based on optical and/or electrostatic models. The traditional resolution of the paradox uses tools outside one-particle relativistic quantum mechanics where particle/anti-particle pair creation is employed. In this paper, we show that the paradox could be resolved within quantum mechanics by including virtual negative energy solutions under the barrier that are usually removed in the conventional treatment. We gauge these negative energy solutions with our own interpretation in which we follow a procedure similar to that in optics where virtual sources are included in the unphysical region to obtain the correct solution in the physical region. An alternative to this interpretation of the negative energy solution is also found in electrostatics

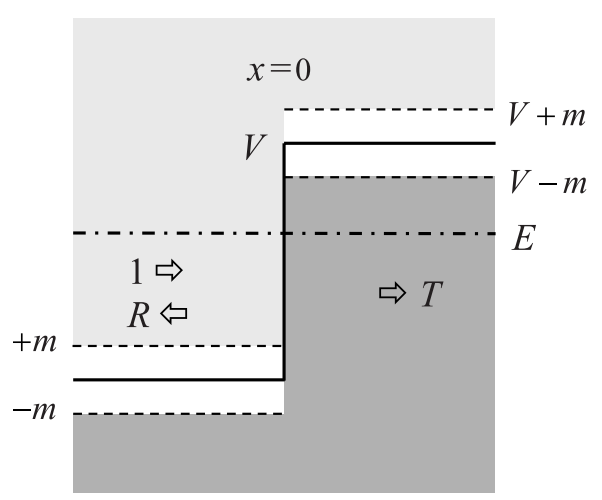

Figure 1. The physical configuration associated with the Klein paradox problem. The height of the potential step is $V>2 \mathrm{~m}$ and the energy is in the range $\mathrm{V}-m>E>+m$. The positive (negative) energy continuum is the region with the lighter (darker) shade. The solid line represents the vector potential $V$ and the dashed lines represent $\pm m$ or $V \pm m$. The energy level is indicated by the dashed-dotted line.

where virtual image charges are added to obtain the correct solution.

Figure 1 shows the configuration associated with the Klein paradox. The typical interpretation is that a beam of electrons with energy in the range $[+m, V-m]$ incident from the left gets partially reflected at the barrier ${ }^{1}$. To account for the electrons transmitted into the barrier, concepts such as 'charged vacuum' and spontaneously produced electron-positron pairs and so on come into play. In most of the earlier attempts at resolving the paradox, principles and tools like these, which come from fields outside relativistic quantum mechanics (e.g. second quantization, quantum field

1 The literature is rich in contributions to this problem. One may consult, for example, [3] and references therein. 


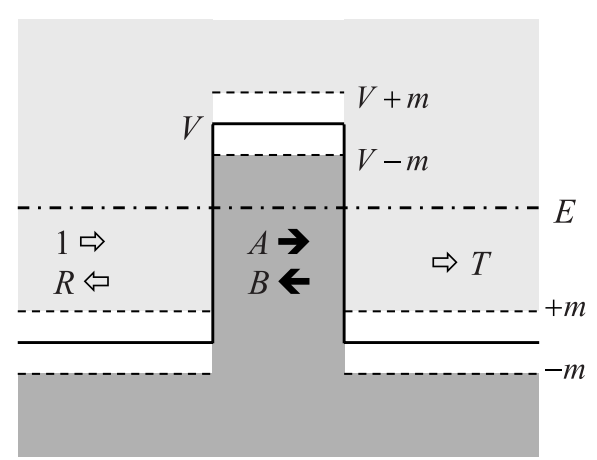

Figure 2. The square barrier problem with the height of the barrier being larger than $2 m$. Electrons are represented by the outlined arrows $(\Longrightarrow)$ and anti-electrons are represented by the solid arrows $(\rightarrow)$.

theory and many particle physics), are deployed. In our opinion, however, a successful resolution of the paradox must come about from within the framework of one-particle relativistic quantum mechanics where the paradox was originally posed. The assertion that the theory at strong coupling is not sufficient for describing this scattering process is debatable since the theory is found to be correct even at higher energies where $E>V-m$ [1]. To address the paradox properly, it helps first to look at the square barrier problem with a particle beam incident from left (see figure 2). The solution of the wave equation to the right of the square barrier consists of positive energy plane waves traveling in the $\pm x$-directions. However, the physical boundary conditions allow only for transmitted waves to the right. On the other hand, in the potential step problem the solution of the Dirac equation to the right of the barrier $(x>0)$ in the Klein energy zone consists of negative energy plane waves traveling in the $\pm x$-directions. Here, however, we will not dismiss the virtual plane wave solutions of negative energy anti-electrons traveling to the left and incident on the barrier. We will utilize this part, which is usually missing from the conventional solution, in the resolution of the paradox and we give it a proper interpretation. The following is a brief technical presentation of the full solution that results in a resolution of the paradox.

In the conventional relativistic units, $\hbar=c=1$, the steady-state Dirac equation for this one-dimensional (1D) problem could be written as follows $[1]^{2}$ :

$$
\left(\begin{array}{cc}
m+V(x)-E & -\frac{\mathrm{d}}{\mathrm{d} x} \\
\frac{\mathrm{d}}{\mathrm{d} x} & -m+V(x)-E
\end{array}\right)\left(\begin{array}{l}
\psi^{+}(x) \\
\psi^{-}(x)
\end{array}\right)=0 .
$$

The potential enters the equation as the time component of a vector with vanishing space component. For $x<0$, where $V(x)=0$, this equation relates the two spinor components as follows:

$$
\psi^{\mp}(x)=\frac{1}{m \pm E} \frac{\mathrm{d}}{\mathrm{d} x} \psi^{ \pm}(x),
$$

which is not valid for $E=\mp m$. We also obtain the following Schrödinger-like second-order differential

2 Multiplying one of the two spinor components by i results in an equivalent representation of the Dirac equation that is identical to equation (1) except that the two off-diagonal elements are replaced by $\mathrm{i} \frac{\mathrm{d}}{\mathrm{d} x}$. equation:

$$
\left(\frac{\mathrm{d}}{\mathrm{d} x^{2}}+E^{2}-m^{2}\right) \psi^{ \pm}(x)=0 .
$$

Now, since $E=\mp m$ belongs to the negative/positive energy spectrum, equations (2) and (3) with the top/bottom sign are valid only for positive/negative energy, respectively. We should emphasize that equation (3) does not give the two components of the spinor that belong to the same energy subspace. One has to choose one sign in equation (3) to obtain only one of the two components and then substitute that into equation (2) with the corresponding sign to obtain the other component. The positive and negative energy subspaces are completely disconnected. This is a general feature of the solution space of the Dirac equation, which is overlooked more often than not. Now, for $x>0$, the same analysis follows but with the substitution $E \rightarrow E-V$.

We start by giving the traditional partial solution of the problem (see footnote 1). The positive energy spinor wavefunction in the Klein energy zone $(m<E<V-m)$ for $x<0$ is

$$
\psi(x)=\frac{1}{\sqrt{1+\alpha^{2}}}\left(\begin{array}{c}
1 \\
\mathrm{i} \alpha
\end{array}\right) \mathrm{e}^{\mathrm{i} k x}+\frac{A}{\sqrt{1+\alpha^{2}}}\left(\begin{array}{c}
1 \\
-\mathrm{i} \alpha
\end{array}\right) \mathrm{e}^{-\mathrm{i} k x},
$$

where $k=\sqrt{E^{2}-m^{2}}$ and $\alpha=\sqrt{(E-m) /(E+m)}$. This solution represents two positive energy electron beams: one beam of unit amplitude incident from the left and another reflected beam of amplitude $A$. For $x>0$, the negative energy solution representing the transmitted beam is

$$
\psi(x)=\frac{B}{\sqrt{1+\beta^{2}}}\left(\begin{array}{c}
-\mathrm{i} \beta \\
1
\end{array}\right) \mathrm{e}^{-\mathrm{i} p x},
$$

where $\quad p=\sqrt{(V-E)^{2}-m^{2}} \quad$ and $\quad \beta=$ $\sqrt{(V-E-m) /(V-E+m)}$. One should note that for positive (negative) energy, $\mathrm{e}^{ \pm \mathrm{i} q x}$ is a wave traveling in the $\pm x$ ( $\mp x$ )-direction, respectively, where $q$ is the positive wave number or linear momentum. Matching the spinor wavefunction at $x=0$ gives

$$
A=(\alpha \beta-1) /(\alpha \beta+1), \quad B=\frac{2 \mathrm{i} \alpha}{\alpha \beta+1} \sqrt{\frac{1+\beta^{2}}{1+\alpha^{2}}} .
$$

The traditional solution stops here with the interpretation that the reflection amplitude is $R=A$ and the transmission amplitude is $T=B \sqrt{\frac{\beta}{\alpha} \frac{1+\alpha^{2}}{1+\beta^{2}}}$. Of course, reality gives $|T|^{2}+$ $|R|^{2}=1$. However, the resulting reflection coefficient, $|R|^{2}$, is less than unity ${ }^{3}$ although the beam energy is less than the height of the barrier. Moreover, the missing electrons penetrating the potential step make the negative energy continuum (vacuum) negatively charged. These two unexpected and paradoxical results were first reported 80 years ago in a classic paper by Klein [2]. The traditional resolution of this paradox is shown in figure 3 [4]. It is interpreted as follows: at the barrier, pair production takes place in which electrons of flux $|T|^{2}$ are reflected to

3 In fact, the value of this reflection coefficient falls within the range $(2 m / V)^{2} \leqslant|R(E)|^{2} \leqslant 1$ with $|R|=1$ at $E=+m$ and $E=V-m$ while $|R|=\frac{2 m}{V}$ at $E=\frac{1}{2} V$. 


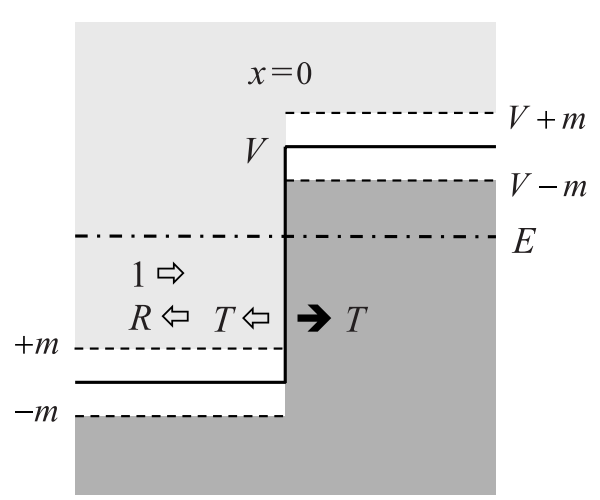

Figure 3. Representation of the traditional resolution of the Klein paradox where spontaneous pair production takes place at the barrier. Positive and negative energy states are created with an equal flux, $|T|^{2}$.

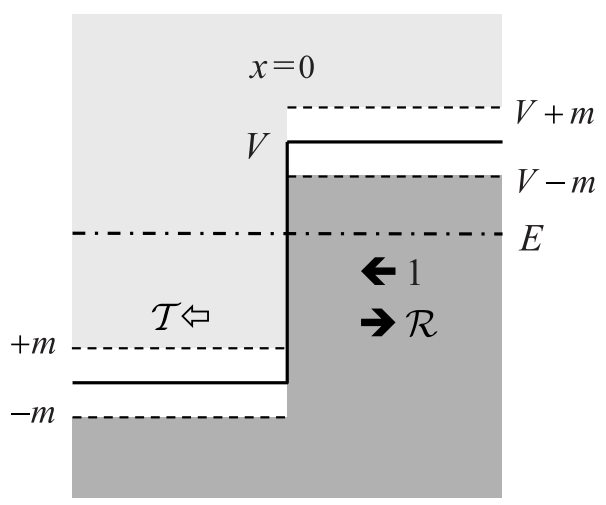

Figure 4. Virtual negative energy incidence is included as a mirror image of the traditional solution. This results in the transmission and reflection coefficients $|\mathcal{T}|^{2}$ and $|\mathcal{R}|^{2}$, respectively.

the left and an equal flux of anti-electrons is transmitted into the barrier. Thus, overall reflection of electrons to the left becomes total since $|T|^{2}+|R|^{2}=1$. Nonetheless, the vacuum becomes positively charged. Moreover, pair production means that one has to use tools and resort to concepts outside one-particle relativistic quantum mechanics where the original problem was presented.

Now, we present our approach to the resolution of the paradox, which is carried out entirely within relativistic quantum mechanics. We do that by including the part of the solution that was eliminated in the conventional treatment. As explained above, it is a plane wave solution of virtual negative energy anti-electrons traveling to the left under the barrier and incident on it. Charge, parity and time (CPT) symmetry of the Dirac equation dictates that we incorporate it as a mirror image of the traditional solution (see figure 4). The mathematical solution of the Dirac equation (1) in the Klein energy zone with the vector potential depicted in figure 1 (without the arrows) could be representing positive energy electrons incident on the barrier from the left, but it could as well be representing negative energy anti-electrons incident on the barrier from the right. It will turn out that the correct solution includes both scenarios while at the same time satisfying the physical boundary conditions. The inclusion of the virtual incident beam is analogous to what is usually done in optics and electrostatics. For example, the following optical model gives a proper interpretation of the negative energy (a)

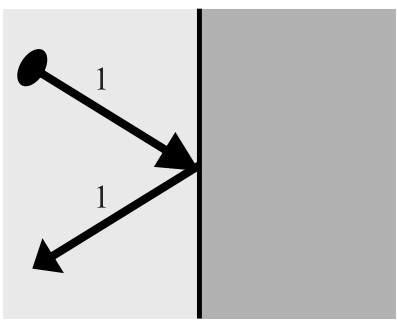

(b)

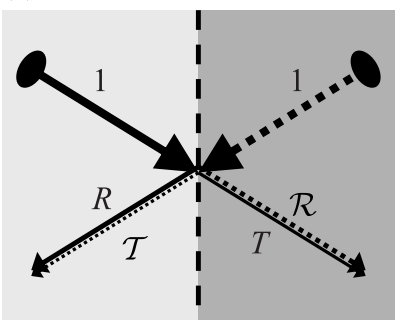

Figure 5. Optical analogue model of the atomic process. (a) The flat mirror located at $x=0$ corresponds to the discontinuity of the vector potential. (b) The region behind the mirror to the right where the imaginary light source is located corresponds to the negative energy continuum.

solution, which is missing from the conventional treatment of the problem: The discontinuity of the vector potential at $x=0$ in the Klein energy zone acts as a perfectly reflecting plane mirror. Figure 5 gives the optical analogue of the atomic process. Incidence and full reflection of light from a light source in front of the plane mirror (left region in figure 5(a)) could be replicated by replacing the mirror (solid vertical line in figure 5(a)) with a partially transmitting plane glass (broken vertical line in figure 5(b)) and placing an identical source of light behind the glass in the unphysical region (see figure $5(\mathrm{~b})$ ). The back of the flat mirror (right region) where the virtual light source is located corresponds to the negative energy continuum. The figure shows the optical beams that correspond to the reflected and transmitted electronic beams $R, \mathcal{R}, T$ and $\mathcal{T}$. It is easy to use symmetry arguments and show that $|R|^{2}+|\mathcal{T}|^{2}=1$. An alternative interpretation is also found in the mirror charges method widely used in electrostatics (see below). These 'mirror models' (optical or electrical) could be used in all problems with such physical configuration in which the vector potential has a discontinuity larger than $2 m$ and the negative energy region extends to infinity.

Therefore, we proceed by including the negative energy solution under the barrier as virtual anti-electrons incident from the right. That is, for $x>0$ it reads as follows:

$$
\chi(x)=\frac{1}{\sqrt{1+\beta^{2}}}\left(\begin{array}{c}
\mathrm{i} \beta \\
1
\end{array}\right) \mathrm{e}^{\mathrm{i} p x}+\frac{C}{\sqrt{1+\beta^{2}}}\left(\begin{array}{c}
-\mathrm{i} \beta \\
1
\end{array}\right) \mathrm{e}^{-\mathrm{i} p x} .
$$

This represents a combination of two negative energy beams of anti-electrons within the potential step. One beam is incident on the barrier from the right with unit amplitude and the other is reflected to the right with an amplitude $C$. For $x<0$, the corresponding solution is

$$
\chi(x)=\frac{D}{\sqrt{1+\alpha^{2}}}\left(\begin{array}{c}
1 \\
-\mathrm{i} \alpha
\end{array}\right) \mathrm{e}^{-\mathrm{i} k x},
$$

which represents a transmitted beam of electrons to the left with amplitude $D$. Continuity of the spinor wavefunction $\chi(x)$ at $x=0$ gives

$$
C=(\alpha \beta-1) /(\alpha \beta+1), \quad D=\frac{2 \mathrm{i} \beta}{\alpha \beta+1} \sqrt{\frac{1+\alpha^{2}}{1+\beta^{2}}} .
$$

Thus, the reflection amplitude in this case is $\mathcal{R}=C$ and the transmission amplitude is $\mathcal{T}=D \sqrt{\frac{\alpha}{\beta} \frac{1+\beta^{2}}{1+\alpha^{2}}}$. Consequently, the 


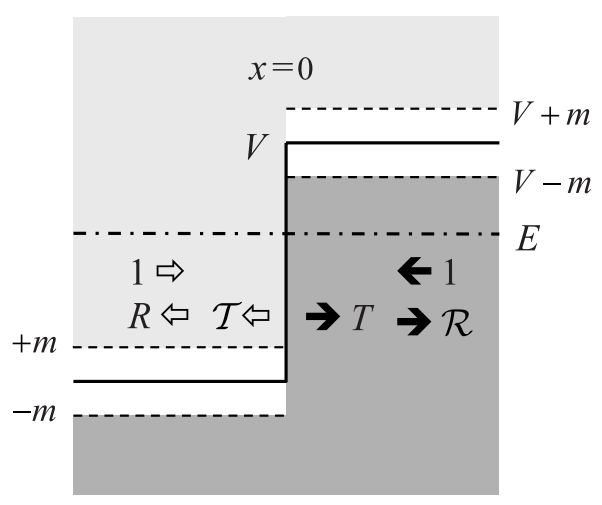

Figure 6. Representation of our proposed resolution of the Klein paradox within one-particle relativistic quantum mechanics (no pair production).

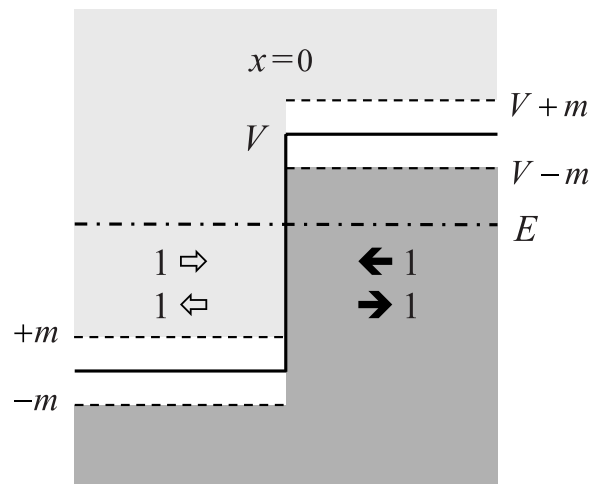

Figure 7. The complete solution of the Klein paradox: (1) total reflection of positive and negative energy waves and (2) particle/anti-particle conservation leaving a neutral vacuum.

observed overall reflection of electrons to the left becomes total since

$$
\left|R^{-}\right|^{2} \equiv|R|^{2}+|\mathcal{T}|^{2}=1
$$

Likewise, the overall reflection of anti-electrons to the right is also total,

$$
\left|R^{+}\right|^{2} \equiv|\mathcal{R}|^{2}+|T|^{2}=1
$$

Figure 6 illustrates the complete process, which is summarized in figure 7 . In the analogous electrostatic problem, positive and negative charges are induced at the boundary. Those with the same sign as the source charge will be displaced to infinity whereas those with the opposite sign will remain at the boundary. The latter are then replaced by the fictitious negative image charge. The process of charge induction at the boundary is equivalent to the process of pair creation at the potential barrier. In analogy, the virtual incident negative energy waves we introduced under the potential step do induce part of the real positive energy reflected waves, which is given by equation (8).

The above results constitute a resolution of the 80-year-old paradox: total reflection without charging the vacuum. The missing part from the traditional solution that we reintroduced is, in fact, a mirror image of the traditional solution. One can also show that this mirror model is equivalent to the boundary condition that the current density, $J(x)$, vanishes at $x=0$, where $J(x)=-\mathrm{i} \psi(x)^{\dagger} \sigma_{3} \sigma_{1} \psi(x)$, $\sigma_{3}=\left(\begin{array}{cc}+1 & 0 \\ 0 & -1\end{array}\right)$ and $\sigma_{1}=\left(\begin{array}{ll}0 & 1 \\ 1 & 0\end{array}\right)$. In general, an incident wave packet, which is sharply centered within the Klein energy zone $(+m<E<V-m)$, will be totally reflected with zero probability of penetrating the potential barrier. However, if the wave packet is sharply centered within the energy range $V \pm m$, then again it will be totally reflected but with non-vanishing probability of barrier penetration. On the other hand, if the wave packet is sharply centered around an energy greater than $V+m$, then partial reflection and transmission will occur. Now, if the energy spectrum of the wave packet extends over all three energy zones, then a linear combination of these scenarios will occur.

Finally, we would like to note that the understanding, interpretation and earlier attempts at a resolution of the Klein paradox presented a few challenges in theoretical physics that lead to significant contributions, which proved to be very enriching and fruitful (see, for example, [5]). Unlike early attempts to resolve the paradox [4], the present one is carried out entirely within one-particle relativistic quantum mechanics where the original paradox was presented. Moreover, the negative energy continuum (vacuum) remains neutral. We believe that the resolution of the paradox offered here might have implications as significant as the original paradox itself.

\section{Acknowledgments}

This work was sponsored by the Saudi Center for Theoretical Physics (SCTP). We acknowledge partial support from King Fahd University of Petroleum and Minerals (KFUPM).

\section{References}

[1] Greiner W 1994 Relativistic Quantum Mechanics: Wave Equations (Berlin: Springer)

Bjorken J D and Drell S D 1964 Relativistic Quantum Mechanics (New York: McGraw-Hill)

[2] Klein O 1929 Z. Phys. 53157

[3] Bosanac S D 2007 J. Phys. A: Math. Theor. 408991 De Leo S and Rotelli P P 2006 Phys. Rev. A 73042107 de Castro A S 2001 Am. J. Phys. 691111

Nitta H, Kudo T and Minowa H 1999 Am. J. Phys. 67966

Calogeracos A and Dombey N 1999 Int. J. Mod. Phys. A 14631

Calogeracos A and Dombey N 1998 Physica B 255126

Su R K, Siu G G and Chou X 1993 J. Phys. A: Math. Gen. 261001

Dominguezadame F 1992 Phys. Lett. A 16218

Evans A B 1991 Found. Phys. 21633

Seba P 1989 Lett. Math. Phys. 1877

Fishbane P M, Gasiorowicz S G, Johannsen D C and Kaus P 1983 Phys. Rev. D27 2433

Bakke F and Wergeland H 1982 Phys. Scr. 25911

Fanchi J R 1981 Found. Phys. 11493

Hansen A and Ravndal F 1981 Phys. Scr. 231036

[4] Sauter F 1931 Z. Phys. 69742

Sauter F 1931 Z. Phys. 73547

Hund F 1940 Z. Phys. 1171

Sommerfeld A 1951 Atombau and Spektrallinien vol II (Braunschweig: Vieweg)

Nikishov A I 1970 Nucl. Phys. B 21346

[5] Holstein R R 1998 Am. J. Phys. 66507

Dombey N and Calogeracos A 1999 Phys. Rep. 31541

Calogeracos A and Dombey N 1999 Contemp. Phys. 40313

Krekora P, Su Q and Grobe R 2004 Phys. Rev. Lett. 92040406

Krekora P, Su Q and Grobe R 2005 Phys. Rev. A 72064103

Katsnelson M I, Novoselov K S and Geim A K 2006 Nat. Phys. 2620

Bai C X and Zhang X D 2007 Phys. Rev. B 76075430 